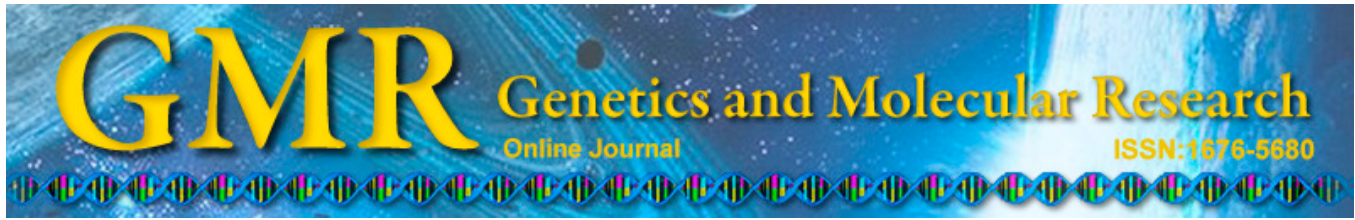

\title{
A comparative study of stress-related gene expression under single stress and intercross stress in rice
}

\author{
Y.P. Zhang ${ }^{1 *}$, Z.G. E ${ }^{1 *}$, H. Jiang ${ }^{2}$, L. Wang ${ }^{1}$, J. Zhou ${ }^{3}$ and D.F. Zhu ${ }^{1}$ \\ ${ }^{1}$ State Key Laboratory of Rice Biology, \\ China National Rice Research Institute, Hangzhou, China \\ ${ }^{2}$ Institute of Plant Protection and Microbiology, \\ Zhejiang Academy of Agricultural Science, Hangzhou, China \\ ${ }^{3}$ St. Laurent Institute, Woburn, MA, USA \\ *These authors contributed equally to this study. \\ Corresponding authors: D.F. Zhu / J. Zhou \\ E-mail: zhudefeng@caas.cn / Jianhua55@msn.com
}

Genet. Mol. Res. 14 (2): 3702-3717 (2015)

Received June 6, 2014

Accepted October 24, 2014

Published April 17, 2015

DOI http://dx.doi.org/10.4238/2015.April.17.20

\begin{abstract}
Plant hormones play important roles in the crosstalk between biotic and abiotic stresses in rice throughout its entire growth period. However, these interactions are not completely understood. In this study, the physiological performance of rice seedlings under a single stress and a sequential combination of various stresses (intercross stress) was determined. We found that catalase, superoxide dismutase, and peroxidase activities and malondialdehyde were highly regulated by intercross stresses. Furthermore, the expression levels of pathogenesisrelated genes and drought stress-related genes under various treatments were analyzed. We demonstrated that under drought-disease intercross stress, the expression levels of the PR4, PAL, and Cht-1 genes were significantly upregulated, while under salt-disease intercross stress, the expression levels of the PRla, PBZ1, Gns 1, and Cht-1 genes underwent significant changes. Regardless of the type of intercross stress, the
\end{abstract}


expression of $L O X-R L L$ was significantly affected. We also showed that the expression of drought stress-related genes OsSKIPa, OsNADPH1, $J R C 0594$, and $O s G L 1-2$ was significantly regulated, suggesting that these genes play important roles in the interaction between biotic and abiotic stresses. We, therefore, conclude that the interactions between various types of biotic and abiotic stresses vary in a complex pattern and would require further in-depth investigation.

Key words: Rice; Abiotic stress; Biotic stress; Hormone

\section{INTRODUCTION}

Due to their immobility, plants are unable to avoid adverse environmental conditions once rooted to the soil. Therefore, plants must possess complete stress response mechanisms to overcome various stresses that may be encountered (Atkinson and Urwin, 2012). Rice is an important food crop, and stability in its yield has a social and economical significance (De Datta, 1981; Khush, 2001). Furthermore, rice is the most important model crop of the Poaceae family. Research on rice has not only increased our understanding of various related mechanisms and helped improve rice varieties but also contributed to knowledge regarding related molecular mechanisms underlying the population improvement of other gramineous crops.

During growth, rice plants encounter a variety of biotic and abiotic stresses. The abiotic stresses mainly include drought, heat, salt, and heavy metals, while the biotic stresses mainly include diseases caused by fungi, bacteria, viruses, and various pests (Yang et al., 2013). Based on the published rice genome and the established genetic transformation system, the effects of single stresses on rice growth and development have been reported extensively. Numerous stress-related genes have also been isolated and analyzed functionally (Jiang et al., 2012) including the $S K C 1$ gene that encodes an HKT-type transporter and maintains $\mathrm{K}^{+}$ homeostasis under salt stress (Ren et al., 2005); the heavy metal stress-related gene OsHMA3 that regulates $\mathrm{Cd}$ transport in the rice root xylem (Ueno et al., 2010); the Bph14 gene (first isolated in rice) that is associated with brown planthopper resistance (Du et al., 2009); the Xa21 gene (first isolated by map-based cloning) that regulates bacterial blight resistance in rice (Song et al., 1995); and several other major resistance genes that regulate resistance to rice blast (Liu et al., 2013). Furthermore, several studies have reported the signal transduction and activation of downstream pathways related to resistance (Hadiarto and Tran, 2011; Derksen et al., 2013). However, rice plants do not experience single stresses during their entire growth process. The stress is usually combinatorial and it has been recognized that there is significant crosstalk between different stresses (Fujita et al., 2006). In addition to a complete stress response system for coping with single stresses, rice plants also require a more powerful regulatory mechanism for multiple stress response systems. Previous studies have suggested that plant hormones play a vital role in regulating a variety of stresses in rice, similar to other plants, e.g., tobacco, Arabidopsis thaliana (Atkinson and Urwin, 2012; Yang et al., 2013). Numerous transcription factors are believed to be involved in such sophisticated regulatory mechanisms (Fujita et al., 2009). However, the understanding of the crosstalk between different stresses in rice is limited.

Plants frequently encounter extreme climates due to the increase in atmospheric pollution. Rice yield is negatively affected by severe drought caused by water scarcity and high 
temperatures (Tirado et al., 2010), as well as by various rice diseases caused by pathogenic bacteria. The occurrence of diseases leads to extensive pesticide usage, further increasing environmental pollution. Rice blast is a destructive disease that occurs in almost all regions of rice cultivation worldwide, causing 11-30\% annual loss in the yield of rice (Wilson and Talbot, 2009). The rice blast fungus Magnaporthe grisea and rice are important models for studying pathogen-host interactions (Dean et al., 2005). However, few studies have been conducted on the interaction between drought and biotic stresses; furthermore, the underlying molecular mechanisms have been rarely reported. Therefore, we conducted intercross stress experiments to investigate the stress response mechanism in rice under a single stress and combined stresses by monitoring changes in the expression levels of related genes. Several studies have shown that drought and salt stresses affect gene expression in a similar manner ( $\mathrm{Li}$ and $\mathrm{Xu}, 2007$; Hadiarto and Tran, 2011). Therefore, this study also investigated the effects of salt stress and salt-disease intercross stress on the expression levels of disease- and drought-related genes.

Because rice is affected by various environmental factors during its life cycle, it is important to closely examine the mechanisms underlying stress resistance and crosstalk between the stresses to promote the study of rice at the molecular biological level. Moreover, this would provide a reference and a theoretical basis for the cultivation of stress-resistant rice varieties.

\section{MATERIAL AND METHODS}

\section{Experimental materials and cultivation of rice seedlings}

The Nipponbare (Oryza sativa L.) rice used in this experiment was kindly provided by Dr. Qian Aian's Laboratory, China National Rice Research Institute, China. Before soaking, the Nipponbare rice seeds were placed in an oven at $43^{\circ} \mathrm{C}$ for 2 weeks to break dormancy. They were then soaked in clear water at $37^{\circ} \mathrm{C}$. The water was replaced daily and poured out after 2 days. Accelerated germination was performed at $37^{\circ} \mathrm{C}$ for 1 day. Well-sprouted seeds were selected and sown on a bottomless $96-$ well plate, which was placed in a hydroponic nutrient solution and then in a light incubator. The light-dark cycle involved illumination for 13 $\mathrm{h}$ at $26^{\circ} \mathrm{C}$ and darkness for $11 \mathrm{~h}$ at $22^{\circ} \mathrm{C}$. The seedlings were cultured to the one-tip three-leaf stage. The hydroponic nutrient solution was prepared and replaced as described previously (Xue et al., 2009).

\section{Disease inoculation, salt stress, and drought stress treatments at the seedling stage}

The Guy11 M. grisea strain inoculated onto the rice seedlings was a gift from the Dr. Lin Fu-Cheng's laboratory, Zhejiang University. The activation and culture of the M. grisea strain was performed according to previously described methods (Valent et al., 1991). The filter paper preserving the strain was inoculated onto oat medium and placed at $26^{\circ} \mathrm{C}$ in a 12:12-h light-dark cycle for activation. On day 3 , when a fresh mycelium emerged at the edge, a small patch of mycelium was inoculated onto a $9-\mathrm{cm}$ solid oat medium and cultured at $26^{\circ} \mathrm{C}$ in a 12:12-h light-dark cycle for 7 days. The spores were washed with $0.2 \%$ gelatin solution, and the spore concentration was adjusted to $5 \times 10^{4} / \mathrm{mL}$. The spore suspension was evenly sprayed on living rice seedlings that were being cultured in alternating light-dark cycles. The inoculated rice seedlings were placed at $24^{\circ} \mathrm{C}$ in the dark for $24 \mathrm{~h}$ for moisturizing treatment and then cultured at $24^{\circ} \mathrm{C}$ and $90 \%$ humidity in a $16: 8$-h light-dark cycle. 
Salt stress treatment was carried out using $\mathrm{NaCl}$ at a final concentration of $140 \mathrm{mM}$ $[0.818 \%(\mathrm{~g} / \mathrm{V})]$ (Ren et al., 2005); drought was simulated using 15\% polyethylene glycol 6000 (Hu et al., 2008).

\section{Determination of physiological and biochemical indicators}

Fresh rice leaf samples $(0.3 \mathrm{~g})$ were weighed and placed in $8 \mathrm{~mL}, 0.05 \mathrm{M}$ phosphatebuffered saline, $\mathrm{pH} 7.8\left(1000 \mathrm{~mL} 8.01 \mathrm{~g} \mathrm{Na}_{2} \mathrm{HPO}_{4} \cdot 12 \mathrm{H}_{2} \mathrm{O}\right.$ and $0.68 \mathrm{~g} \mathrm{KH}_{2} \mathrm{PO}_{4}$ solution) for homogenization in an ice bath. The homogenized sample was placed in a $15-\mathrm{mL}$ centrifuge tube, and centrifugation was carried out for $20 \mathrm{~min}$ at $4^{\circ} \mathrm{C}$ and $10,000 \mathrm{~g}$. The supernatant was used for analysis of catalase (CAT), superoxide dismutase (SOD), and peroxidase (POD) activities, and malondialdehyde (MDA) content. This process was repeated 3 times.

The spectrophotometric thiobarbituric acid reactive substance test was used to determine the MDA content. SOD activity was determined by monitoring the photochemical reduction of nitroblue tetrazolium; POD activity was determined by the guaiacol oxidation method; and CAT activity was measured by the ultraviolet absorption method. The absorbance was measured using a SHIMADZU UV-2410PC spectrophotometer and an enzyme kinetics software bundled with the equipment that was manufactured in Japan.

\section{RNA extraction and quantitative fluorescence analysis}

RNA extraction was performed using the aboveground part of rice seedlings in the control group and those treated with different stresses. RNA was extracted using TRIzol reagent (Invitrogen) according to manufacturer instructions. Reverse transcription of total RNA was carried out using the DNase digestion kit (Invitrogen). First-strand cDNA was obtained by reverse transcription using AMV reverse transcriptase (TaKaRa) according to manufacturer instructions. The products of reverse transcription were stored at $-80^{\circ} \mathrm{C}$ until further analysis. Quantitative fluorescence experiments were performed using the ABI 7500 Real-Time PCR instrument. Relative gene expression levels were calculated using the $2^{-\Delta \Delta \mathrm{Ct}}$ method (Livak and Schmittgen, 2001) with the following equations:

$$
\begin{aligned}
\Delta \mathrm{C}_{\mathrm{T} \text { (test) }}=\mathrm{C}_{\mathrm{T} \text { (target, test) }}-\mathrm{C}_{\mathrm{T} \text { (ref, test) }} & \text { (Equation 1) } \\
\Delta \mathrm{C}_{\mathrm{T} \text { (calibrator) }}=\mathrm{C}_{\mathrm{T} \text { (target, calibrator) }}-\mathrm{C}_{\mathrm{T} \text { (ref, calibrator) }} & \text { (Equation 2) } \\
\Delta \Delta \mathrm{C}_{\mathrm{T}}=\Delta \mathrm{C}_{\mathrm{T} \text { (test) }}-\Delta \mathrm{C}_{\mathrm{T} \text { (calibrator) }} & \text { (Equation 3) }
\end{aligned}
$$

The final relative expression level of an individual gene was $2^{-\Delta \Delta C t}$.

\section{Data processing and charting}

The Statistical Package for the Social Sciences (SPSS, version 20, IBM, USA) was used for the statistical analysis of data from physiological and quantitative fluorescence experiments. The lattice package in R programming language (version 2.15.3, USA) was used to prepare the line chart of the expression of resistance-related genes, and the "pheatmap" package in $\mathrm{R}$ was used to plot the heat map for quantitative analysis. 


\section{Microarray data verification and analysis}

Analysis of microarray expression data was performed using dChip (5/12, 2011 software, Harvard University, USA) (Li, 2008). The microarray data were obtained from National Center for Biotechnology Information Gene Expression Omnibus (GEO) under the following GEO accession Nos.: GSE24048 for microarray of different varieties that experienced drought; GSE26280 for gene expression analysis of different rice tissues under drought stress (Wang et al., 2011); GSE21651 for gene expression analysis of varieties that had different sensitivities to drought and salt stresses; GSE7256 for long-term gene expression analysis of rice inoculated with M. grisea (Ribot et al., 2008); GSE30941 for gene expression analysis of rice inoculated with different strains (Abbruscato et al., 2012); GSE41798 for gene expression analysis of different inoculated rice varieties (Wei et al., 2013); and GSE6901 for gene expression analysis of rice under drought, salt, and cold stresses (Sharma et al., 2009). The CEL documents of all experiments were first downloaded from the database, and data reading, homogenization, and cluster analysis were then performed using the dChip software; the heat map was finally generated.

\section{RESULTS}

\section{Physiological performance of rice plants under single and intercross stresses}

In this study, different combinations of stresses were selected to analyze the interaction between various stresses (Figure 1).

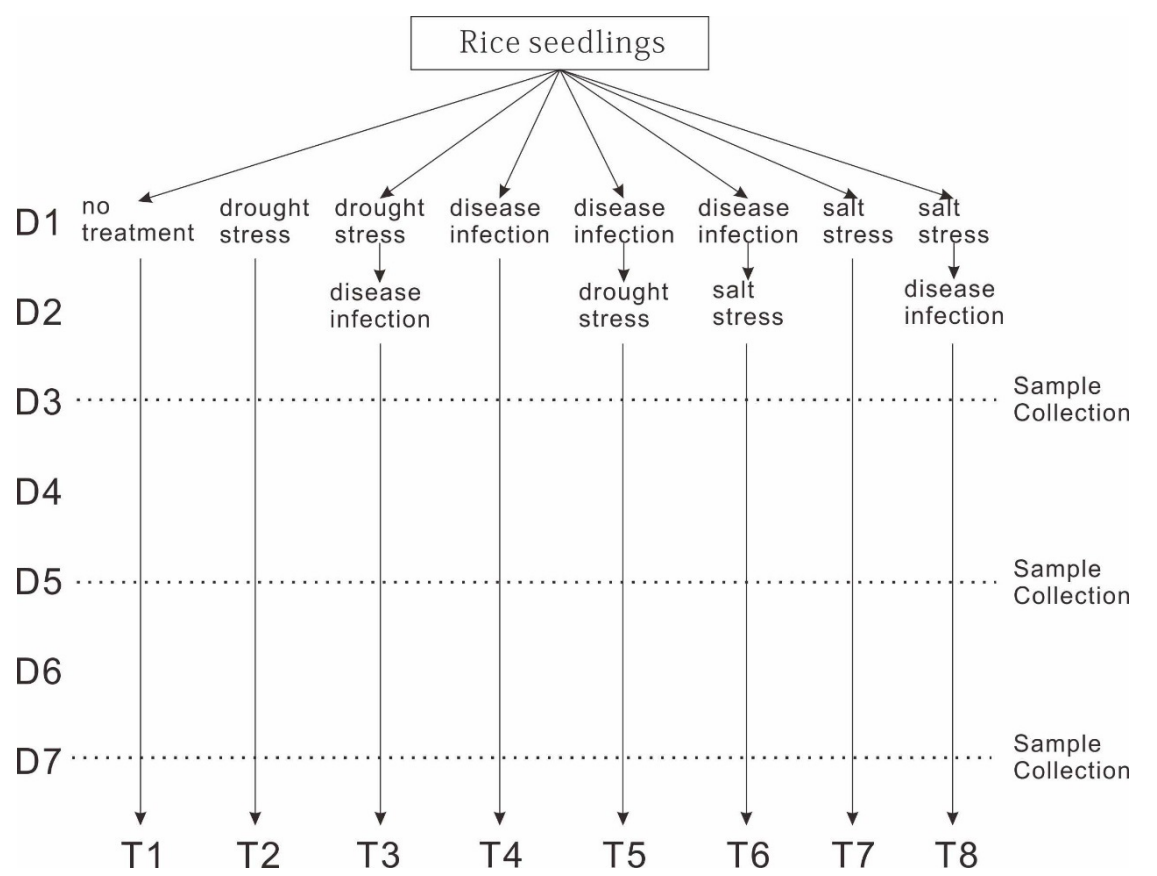

Figure 1. Diagram of experimental design and sampling time. D1-D7 correspond to experimental days. T1-T7 correspond to experimental treatments. 
Because it generally takes $\sim 24 \mathrm{~h}$ to observe the effects of bacterial inoculation (Bagnaresi et al., 2012), the first sampling point was set on day 1 after inoculation, i.e., on day 3 after treatment. Sampling was performed 3 times, once every other day (Figure 1).

All results are reported as percentages of physiological indicators of the test plants relative to the control plants (Figure 2 and $\underline{\text { Figure S1) }}$ ).

A
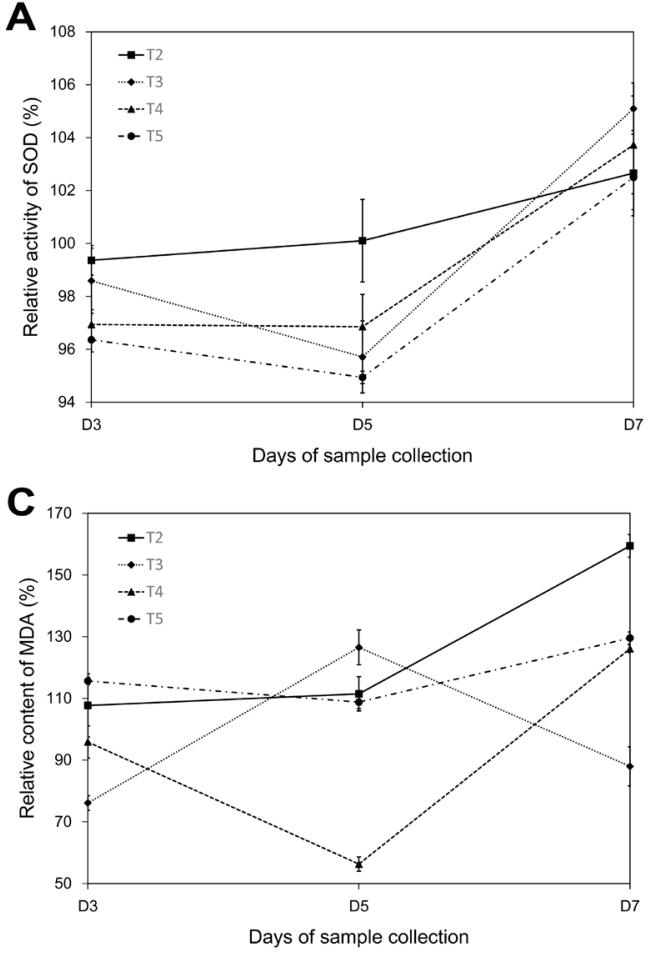
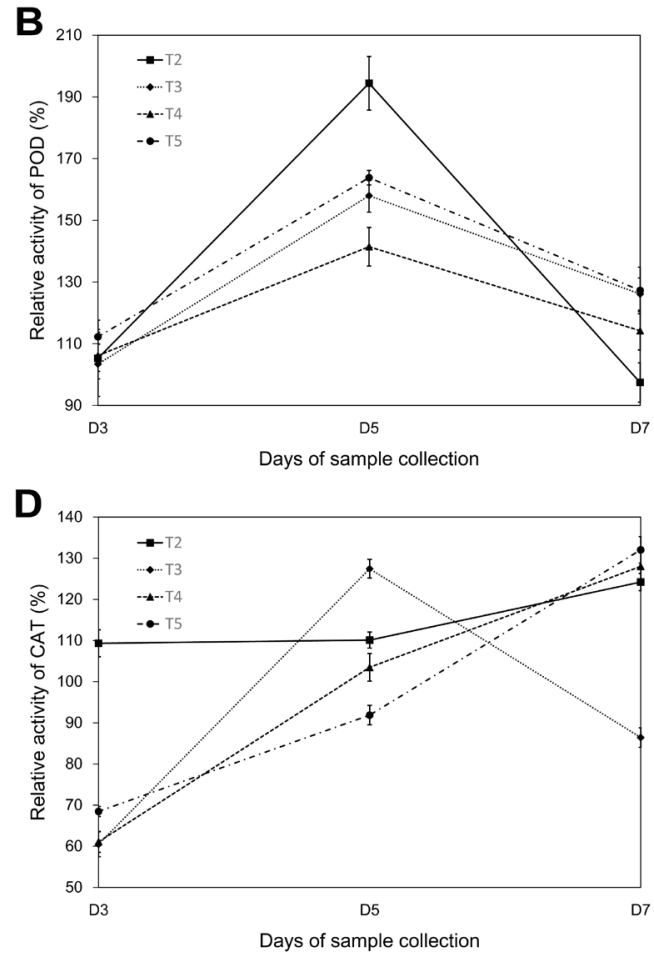

Figure 2. Relative values of various physiological indicators under different treatment conditions. A. Relative activity of superoxide dismutase (SOD). B. Relative activity of peroxidase (POD). C. Relative content of malondialdehyde (MDA). D. Relative activity of catalase (CAT). T2, T3, T4, and T5 correspond to different treatments indicated in Figure 1.

The results showed that under a single stress of drought or disease, the SOD activity increased with treatment time (Figure 2A). However, under salt stress, the SOD activity initially decreased and then increased (Figure S1A). Under the intercross stress of drought and disease, the SOD activity initially decreased and then increased. Under salt-disease intercross stress, the SOD activity initially increased and then decreased or increased continuously when the disease treatment was performed before or after salt stress. In the intercross stress, the final total SOD activity was always higher when the rice plants were first subjected to abiotic stress than when the plants were subjected to a single stress. However, if the plants were first subjected to disease, the final SOD activity decreased, regardless of the type of the subsequent stress.

The POD activity initially increased and then decreased under all treatments at all sam- 
pling time points, except under the T6 treatment when the POD activity continuously increased. Unlike the SOD activity, the final POD activity was not related to the sequence of the stresses, and it increased with an increase in the number of types of stresses (Figure 2B and Figure S1B).

The change in the MDA content was similar to that in the SOD activity, initially increasing and then decreasing under the T3 treatment. The MDA contents under the intercross stresses of disease and other stress types were lower than those under single stresses, except under the $\mathrm{T} 6$ treatment (Figure 2C and Figure S1C).

The CAT activity under the $\mathrm{T} 3$ treatment showed a variable trend similar to the trend for the MDA content. All CAT activities, except that under single salt stress, increased with time (Figure 2D and Figure S1D). Similar to the SOD activity and MDA content, all CAT activities under disease intercross stresses and other stress types were lower than those under single stresses.

\section{Expression of pathogenesis-related genes in rice}

To understand the expression of pathogenesis-related genes under various stresses, 10 pathogenesis-related genes were selected for quantitative fluorescence analysis under different treatments (Table 1 and $\underline{\text { Table S1). }}$.

Table 1. Genes selected in this study and the primers for quantification.

\begin{tabular}{|c|c|c|}
\hline Gene name & Accession No. & Sequence (5'-3') \\
\hline PRIa & AJ278436 & GGTGTCGGAGAAGCAGTGGTA; GCGAGTAGTTGCAGGTGATGAAG \\
\hline$P R I b$ & U89895 & AGGCGTTCGCGGAGAACTA; GAAGAGGTTCTCGCCAAGGTT \\
\hline PR4 & AY050642 & CATTATTACAACCCACAACAGAACAA; GCATCCCATGTGGCACAAT \\
\hline$P B Z 1$ & AF274850 & GGCTTGGTCGACGACATTG; CAGGGTTAAGCTTCATGGTGTAGA \\
\hline JIOsPRIO & AF395880 & GCAGCGTCAGGCAGTTCAA; GAACTCCAGCCTCTCCTTCATG \\
\hline Gnsl & X58877 & GGCGTCGAGCAGAATTGG; CGGAATGCATCAGAAGCTGAT \\
\hline Cht -1 & D16221 & GGCGTTCTGGTTCTGGATGAC; CGCCGTTGATGATGTTGGTC \\
\hline$P A L$ & X16099 & GGTGTTCTGCGAGGTGATGA; AGGGTGGTGCTTCAGCTTGT \\
\hline CHS & AB000801 & CCGGCGAACTGCGTGTAC; CACATCCTCTTGAACTTCTCCTTGA \\
\hline$L O X-R L L$ & D14000 & AGATGAGGCGCGTGATGAC; CATGGAAGTCGAGCATGAACA \\
\hline OsPIPI-3 & AB029325 & TGGACGGAAAGATTATGTCTGA; CCCCTGTGTGTCGTGTAGTG \\
\hline OsGL1-2 & AK066569 & CACCCTCAACCACAAGTCAT; TGCCAGGAACACGAAGTC \\
\hline OSLEA3-1 & DQ789359 & GCGAGTGAGCAGGTGAAGA; GTGGCAGAGGTGTCCTTGTT \\
\hline OsDHNI & AY786415 & CGGATGGGAAGGAGAAGAAG; CAAACTAACACACGCCAAACA \\
\hline OsMTIa & OSU46159 & TTCTCACTGTCATCTATCCTCTCTG; CAACCTTTTCCATCATCCTATG \\
\hline OsDHODHI & DQ102485 & GCCTCTCTCCCGTATTTCAC; CGGTCCAGTCCTTGTCTGAT \\
\hline OsNADPHI & AB246888 & CTGATGGCTGATGCTGTGTT; GACCTGGCGTTCTTGTGAG \\
\hline$O C P I 1$ & AK062495 & GGACAATAACACCCGTTTCAGT; GGCTTGCTGTCTGATGAACTC \\
\hline OsSKIPa & EU368691 & GGGCGTGACAGATTGGATTA; GCCGATTGAAGAACAAAAGGTTAC \\
\hline OSDREB $2 B$ & AK099221 & ATCCACAGGGTCCAAAGAAG; CACACCACGGAAGTCACAAC \\
\hline Osbzip23 & AK072062 & CACATCССАССТСТССТСАG; CТСАСССААССАААССААТС \\
\hline snacl & DQ394702 & АААТСССТСАСААСССАСАА; СТСАТССССАТСGСТTСТТ \\
\hline Snac2 & AK068392 & AGGGCGAGAAGACCAACTG; ACCCAATCATCCAACCTGAG \\
\hline OsWRKY72 & BK005075 & CGAGAAATCCAACGACAACTT; GCTGAAGGGAAGAGAGGTGAG \\
\hline OSTIFY11a & AK070649 & GTCGAGTTGGAAGATGGTTCTC; CACCCCTCTCTAGTCCTGATCT \\
\hline OSMPK5 & AF479883 & GGAGATGGTGGCGATAAAGA; TCCCTGATGCCTATGATGTTC \\
\hline DSM1 & AK102767 & GGGGTCATACTGTGGGAACTT; TATCAAGGCGACGATTCTGA \\
\hline OsSIK1 & AK064052 & CTCGCATAATCCACAGAGATG; TGGCAGAGGGGACACATT \\
\hline OsCDPK7 & $\mathrm{AB} 042550$ & CGGACGAAGAACAATGAGAA; GGCAGGAAGACAGGAAGAAG \\
\hline OsTPPl & AB120515 & TTCTGCTTTGGCTTCCTTCA; TCATCCACAATAGGCGACAG \\
\hline OsRR6 & AJ938075 & GTCCCCAACGTCAACATGA; TCTCCTTGAGCTGAGACGATTC \\
\hline$O S A O X 1 \mathrm{a}$ & AK064040 & GATGTTTGTCTACTGCCGAGGATTT; ATGTTAGTATATATAACTCAGCTGCC \\
\hline OsAldh $2 \mathrm{a}$ & AK121610 & TAGCTGTAGTAATCGATC; TGTACAAAAGATTGCCCG \\
\hline$O s P P 2 \mathrm{C}$ & AK071637 & GGAGGCACTTCTATGACACC; AGAAGTTCAGAGTCCGTGCT \\
\hline JRC0549 & AK105513 & TCAGGATTCCAGTCCAAGCA; CGTTGGTGTGCAGCGTGTAC \\
\hline OsSUT & AK099306 & GTGGCGCAGGAGGTGAAGCA; CCCCACCCTGTCCACCGAGT \\
\hline actin & AK100267 & GAGCTACGAGCTTCCTGATGGA; CCTCAGGGCAGCGGAAA \\
\hline
\end{tabular}


The results of the analysis showed that the expression of these genes varied across the treatments (Figure 3).

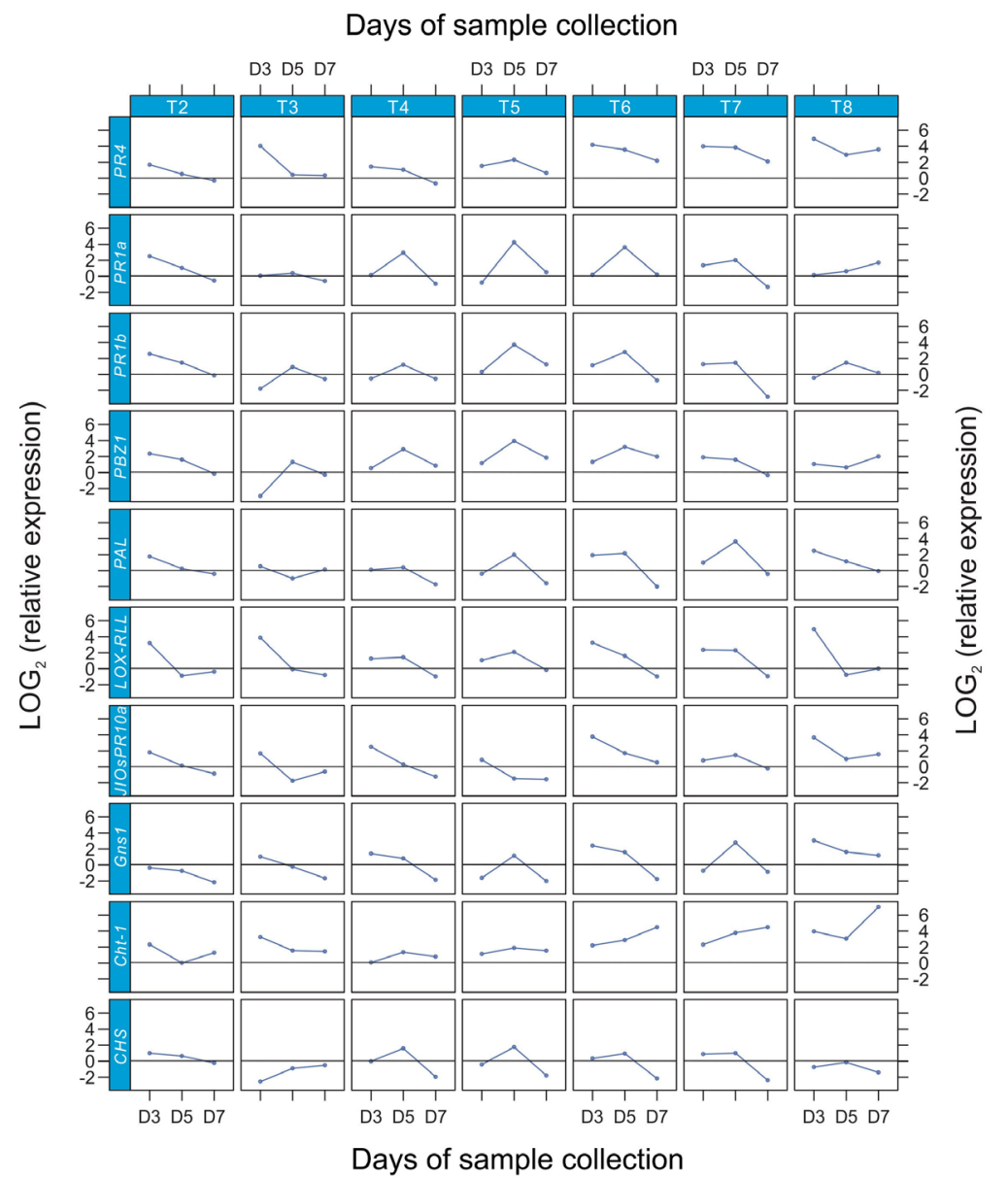

Figure 3. Levels of expression of various resistance-related genes under different treatments. T2, T3, T4, T5, T6, $\mathrm{T} 7$, and T8 correspond to various treatments and D3, D5, and D7 correspond to sampling times indicated in Figure 1 ; the lines represent the relative expression levels of various pathogenesis-related genes.

Most of the pathogenesis-related genes were upregulated under single drought stress. The expression of these genes was downregulated under prolonged stress. The expression of most of the pathogenesis-related genes was not significantly upregulated under single disease stress. However, the expression of the genes was significantly upregulated in D5 samples and then downregulated at later stages. The expression of the pathogenesis-related genes under single salt stress was similar to their expression under single drought stress, although there was an intense downregulation in the later stages. Under intercross stresses in which the plants were subject to disease stress first, there was no significant change in the expression levels of the various pathogenesis-related genes, although the expression levels during each period 
changed to some extent. In contrast, when disease stress was secondary, there were significant changes in the expression levels of some pathogenesis-related genes. Under drought-disease intercross stress, the expression levels of the PR4, PAL, and Cht-1 genes were significantly upregulated. Under salt-disease intercross stress, the expression levels of PRIa, PBZ1, Gns , and $C h t-1$ genes underwent significant changes. Regardless of the type of intercross stress, the expression of $L O X-R L L$ was significantly affected.

\section{Analysis of drought stress-related gene expression}

Drought stress-related gene expression was analyzed in addition to pathogenesis-related gene expression. The expression levels of the various genes underwent different changes under prolonged stress (Figure 4).

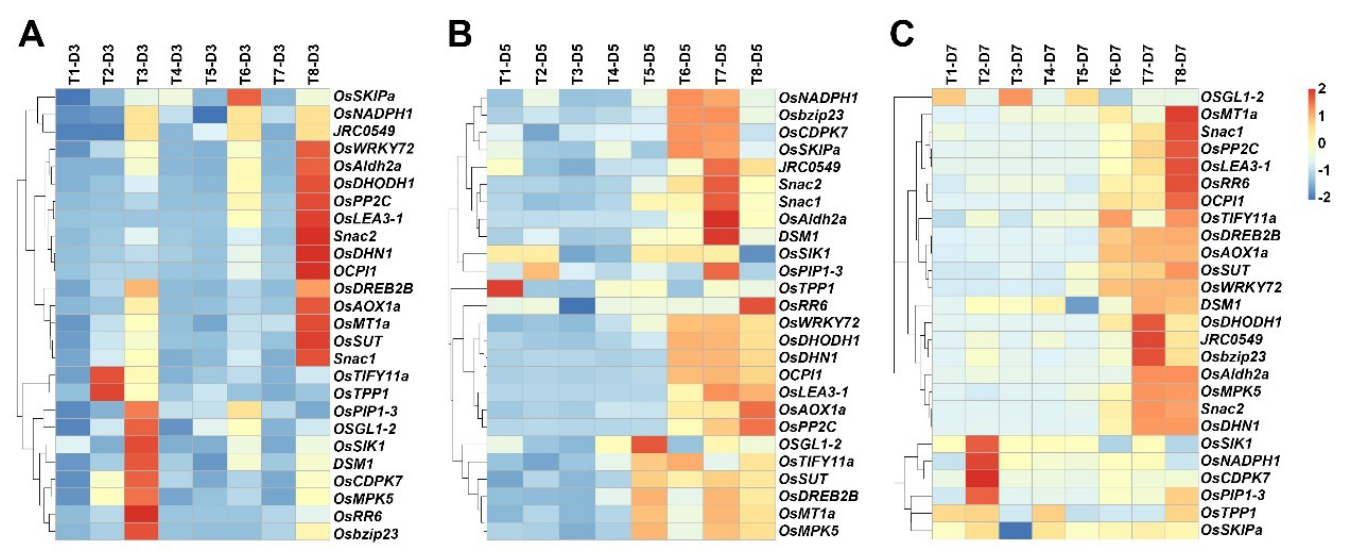

Figure 4. Heat maps of gene expression of various drought stress-related genes under different treatment conditions at different sampling time points. A. First sampling point. B. Second sampling point. C. Third sampling point.

Under single disease stress, the expression levels of drought stress-related genes did not undergo significant changes compared with the control group, regardless of the duration of stress. When the plants were subjected to disease stress first, the following drought stress upregulated the expression of some drought stress-related genes associated with signal transduction and transcription at D5. Moreover, when disease stress was followed by salt stress, the expression levels of most genes were higher than those of genes under a single stress. At D3, the expression levels of most genes under intercross stress in which drought stress occurred first were higher than those of genes under a single stress. Gene expression was upregulated more significantly under the intercross stress in which salt stress occurred first than under a single stress. With a prolonged stress duration, the expression of most genes under intercross stresses in which drought stress occurred first was downregulated, although only to a small degree. Under intercross stresses in which salt stress occurred first, the expression of the genes initially increased, then decreased, and finally increased again. Furthermore, under salt-disease intercross stress, the final levels of gene expression were essentially the same, regardless of the sequence of stresses. Among these genes, the expression levels of the OsSKIPa, OsNADPH1, JRC0594, OsGL1-2, OsPPI1-3, OsTPP1, and DSM1 genes significantly changed with the sampling period and stress treatments, suggesting that these genes are closely associated with 
intercross stress (Figure S2). Except these genes, the genes influenced by drought-disease intercross stress were greatly different from those influenced by salt-disease intercross stress, suggesting that the crosstalk between the 2 stresses with disease was not identical.

\section{Microarray data validation}

To more accurately understand the expression of these genes under various stress conditions, the differences in the expression of the above genes were also analyzed in this study using a microarray (Tables S2, S3 $)$.

The expression levels of various resistant genes varied between the rice varieties, suggesting that the action of these pathogenesis-related genes is dependent on the genetic background (Figure 5A).

A

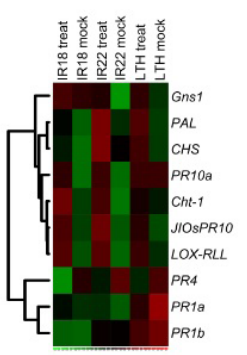

$\mathrm{D}$

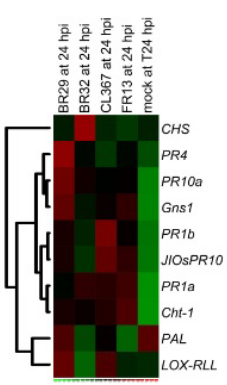

B

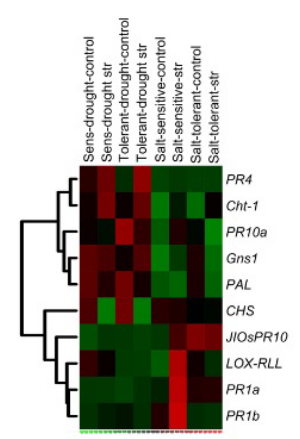

E

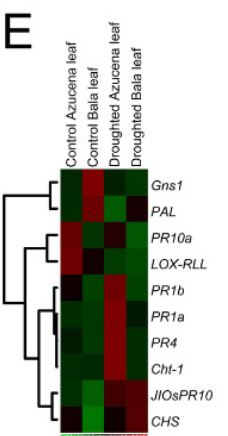

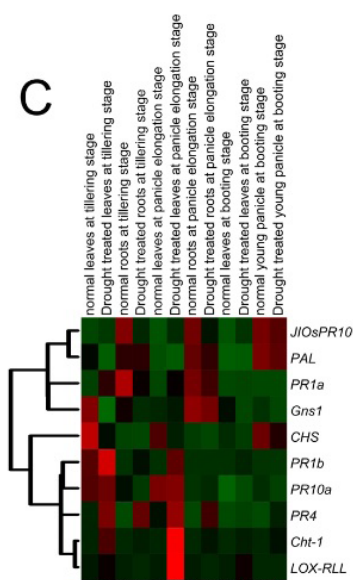

G

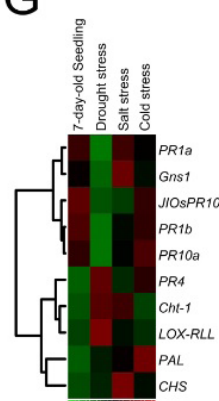

Figure 5. Microarray gene expression analysis of various pathogenesis-related genes in A. different rice varieties under bacterial inoculation, GSE41798; B. different sensitive varieties under drought and salt stress conditions, GSE21651; C. different tissues under drought stress, GSE26280; D. rice plants inoculated with different pathogens, GSE30941; E. different upland rice varieties under drought stress, GSE24048; F. different sampling time points after inoculation of rice pathogens, GSE7256; and G. different stress treatments, GSE6901.

Further, various strains of $M$. grisea had different impacts on the expression of these genes (Figure 5D), and the results of microarray analysis in long-term sampling experiments 
showed an increase in the expression levels of some genes with time. These findings were consistent with the results of quantitative fluorescence analysis, which indicated that the expression levels of the pathogenesis-related genes were higher at D5 (Figure 5F). Drought treatment in various sensitive varieties indicated a significant change in the expression levels of the $P R 4, P A L, C h t-1$, and $L O X-R L L$ genes, and this was consistent with the results of quantitative fluorescence analysis (Figure 5B). However, the expression levels of the pathogenesis-related genes in the upland rice variety Bala did not change significantly, and the results in the upland rice variety Azucena were consistent with the results of the above experiment (Figure 5E), suggesting the existence of more complex crosstalk mechanisms in upland rice. Gene expression analysis in different tissues of rice suggested that the pathogenesis-related genes under drought stress had different expression patterns in various tissues, which was consistent with the results of quantitative fluorescence analysis. The expression levels of the $P R 4, P A L$, and $C h t-1$ genes in the leaves changed significantly; however, completely contrasting results were obtained for the expression in the roots and spikes, suggesting that the interactions are complex in different periods and tissues. Expression analysis of the pathogenesis-related genes under different stresses showed that the roles of these genes varied under different stresses (Figure 5G).

Microarray expression analysis of drought stress-related genes showed that the relevant genes in different varieties under disease stress had similar expression patterns (Figure 6A).

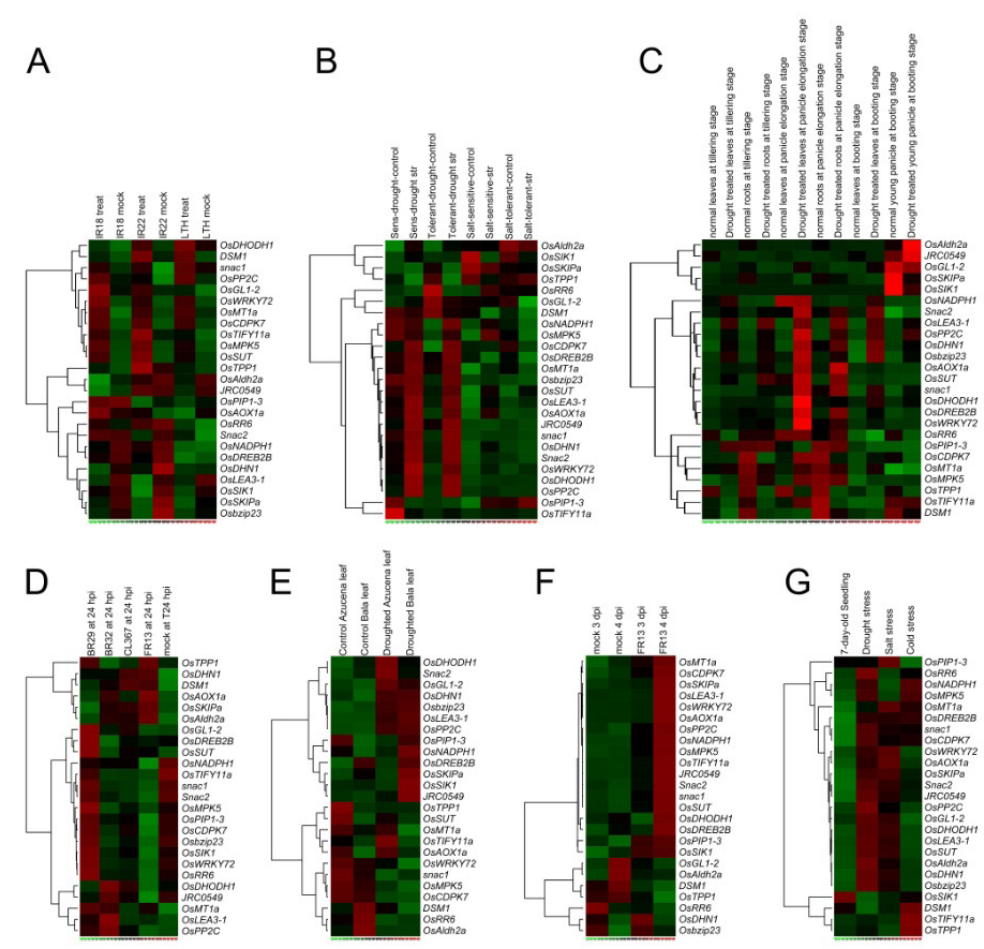

Figure 6. Microarray gene expression analysis of various drought stress-related genes in A. different rice varieties under inoculation, GSE41798; B. different sensitive varieties under drought and salt stress conditions, GSE21651; C. different tissues under drought stress, GSE26280; D. rice plants inoculated with different pathogens, GSE30941; E. different upland rice varieties under drought stress, GSE24048; F. different sampling time points after inoculation of rice pathogens, GSE7256; and G. different stress treatments, GSE6901. 
However, inoculation of different pathogens resulted in completely different expression levels (Figure 6D). The expression levels of drought stress-related genes generally increased over time after disease inoculation (Figure 6F). The expression levels of the genes under drought stress and salt stress were generally consistent, but they were inconsistent with those under other stresses such as cold (Figure 6G). The expression levels of these drought stress-related genes varied between different upland rice varieties (Figure 6E). The expression levels were consistent in various varieties (Figure 6B) but were markedly different in various tissues (Figure 6C).

\section{DISCUSSION}

Rice is an important food crop that is a sufficient source of nutrition for half of the global population (Khush, 2001). Rice plants inevitably encounter a variety of stresses, ranging from adverse environmental conditions to harmful organisms, during their growth process. Unlike animals, plants are incapable of locomotion. Therefore, they use large amounts of energy to actively defend themselves from adverse conditions (Atkinson and Urwin, 2012). This active response to adversity in plants manifests as complex stress responses and regulatory mechanisms at the molecular level (Yang et al., 2013). Furthermore, these sophisticated regulatory mechanisms ultimately lead to other related reactions, such as a variety of physiological responses in rice, thereby helping the plant to survive the stress period.

Of the 4 physiological indicators measured in the present study, SOD activity did not vary significantly between the different treatments. However, the other 3 physiological indicators underwent very significant changes. Furthermore, the changes were specific for the treatments. In plants, the activities of antioxidant enzymes, including SOD and POD, play important roles in the maintenance of membrane integrity and defense against the attack of reactive oxygen species on the membrane (Baisak et al., 1994). The combined action of SOD, POD, and CAT can help maintain a low concentration of free radicals to avoid membrane damage. Plant organs may undergo membrane lipid peroxidation under stress conditions. MDA, which is the end product of membrane lipid peroxidation, is an important indicator of damage to the membrane system and can be used to determine the extent of membrane lipid peroxidation in the cells and the strength of the plant response to adverse conditions. In this study, the changes in the SOD, POD, and CAT activities and MDA content were not identical across the treatments. This suggests that the rice plant uses complex mechanisms in response to biotic and abiotic stresses; however, more in-depth research is necessary.

Few studies have been conducted on the interaction between rice blast and drought stress. Because the invasion by pathogens requires appropriate humidity, the risk of incidence of rice blast under drought conditions is relatively low. Using protein analysis, Yang et al. (2011) identified differentially expressed proteins of pathogenesis-related genes in the roots of rice plants under drought stress, thus indicating that pathogenesis-related genes also play important roles in the response to drought stress. Studies of rice show that several plant hormone pathways, typically the salicylic acid (SA) and jasmonic acid (JA) pathways, are involved in the response to blast disease stress. Of the 10 pathogenesis-related genes selected in our study, the PRIa, PAL, Cht-1, and PR4 genes are primarily involved in the SA pathway; the JIOSPRIO and $L O X-R L L$ genes are primarily associated with the JA pathway; the $P R 1 b$ and $P B Z 1$ genes are associated with the SA and JA pathways (Shen et al., 2011); and the CHS and Gns 1 genes may be associated with the JA pathway. In our study, the pathogenesis-related genes had dif- 
ferent expressions under various conditions of pathogen infection (Figure 5D) and in different varieties (Figure 5A) (Peng et al., 2012). Further, these pathogenesis-related genes had similar expressions in rice varieties with different sensitivities to drought stress (Figure 5B) but different expressions in various tissues (Figure 5C). The gene expression patterns in upland rice varieties were more complex (Figure 5E) and varied under different stresses (Figure 5G). These results suggested the complexity of the regulation of pathogenesis-related genes. However, the active $P R 4, P A L, C h t-1$, and $L O X$-RLL genes under drought-disease intercross stress were also sensitive to single drought stress (Figures 3 and $5 \mathrm{~B}$ ). Therefore, these pathogenesis-related genes may be closely associated with the response to drought and disease and the crosstalk between the 2 stresses. However, biotic stress itself is very complex. The expression levels of various pathogenesis-related genes not only depend on the genetic background (Figure 5A) but also depend on the race of $M$. grisea infecting the rice (Figure 5D). These findings suggested that more influential factors should be considered while studying biotic and abiotic stresses and that the underlying mechanisms are complex.

Drought resistance of rice involves complex mechanisms, including drought escape, drought avoidance, drought tolerance, and drought recovery (Nguyen et al., 1997; Hadiarto and Tran, 2011). Several experimental techniques have been used to identify and investigate drought stress-related genes and pathways. The related genes have been isolated and used in functional studies (Hu et al., 2008; Rabello et al., 2008; Yang et al., 2009; Golldack et al., 2011). To better understand the effects of drought stress-related genes, Yang et al. (2010) divided droughtresponsive genes into 3 categories, namely transcriptional regulation, post-transcriptional modification, and osmoprotectant metabolism or molecular chaperone, on the basis of their biological functions. In this study, a total of 26 drought stress-related genes were included in quantitative fluorescence analysis. Seven of these genes were involved in transcriptional regulation, 5 were involved in post-transcriptional modification, and the remaining 13 were involved in osmoprotectant metabolism or molecular chaperone (Table S1). Analysis of the expression of the genes under various treatment conditions showed that among these genes, the OsSKIPa, OsNADPH1, and JRC0594 genes were not only related to drought-salt stress but also showed significant changes in their expression under disease stress. In addition, some genes were active under intercross stress, which suggests that these genes, including the pathogenesis-related genes (e.g., $P R 4$ ), played important roles in the crosstalk between biotic and abiotic stresses. In this study, of the genes that were identified to be closely related to the interaction between different stresses, only the DSM1 gene belonged to the category of post-transcriptional modification. All other genes belonged to the category of osmoprotectant metabolism or molecular chaperone. The genes related to transcriptional regulation did not undergo significant changes in their expression levels. These results suggested that although there was crosstalk between different stresses, the interactions were concentrated mainly downstream of the signaling pathways, and the various stresses acted via independent signaling pathways (Yang et al., 2013).

Upland rice is an ecotype of rice adapted to conditions of water scarcity (Luo, 2010). Upland rice varieties in different regions have originated from Indica and Japonica rice, both of which show excellent tolerance to drought conditions (Gupta and O'Toole, 1986). The results of microarray analysis showed that different upland rice varieties manifest different mechanisms in drought tolerance (Figure 6E). The expression levels of drought stress-related genes varied significantly with rice varieties (Figure 6A), and the expression levels of the genes under disease stress were considerably different between the upland rice varieties (Figure 5E). Thus, different ecotypes or extreme varieties may behave very differently with regard 
to the crosstalk between various stresses, highlighting the need for separate studies for each ecotype or variety.

Although the phenotypic response of rice to salt stress is different from that to drought stress, bioinformatic analysis and several experiments showed that the expression of salt stressrelated genes and the corresponding pathways were similar to those of drought stress-related genes (Hadiarto and Tran, 2011). Thus, in the study of genes under drought stress, the response to salt stress was also investigated. The effect of drought stress combined with salt stress has been well documented in the literature (Golldack et al., 2011). In the present study, microarray analysis showed that drought and salt stresses were relatively similar in their effects on the expression of related genes (Figure 6G), although their effects in extreme varieties were different (Figure 6B). Furthermore, the difference was more significant when disease stress was involved (Figure S1 and Figure 3). These results indicated that the crosstalk between different stresses is not exactly the same (Figure $5 \mathrm{G}$ ) and that a more complex regulatory mechanism could be involved in the process.

There have been several studies on single biotic or abiotic stresses. It is also known that some common signaling pathways and some interactions exist between abiotic and biotic stresses, although the interactions between them have not been extensively reported. In this context, the present study designed different treatments by combining disease stress, drought stress, and salt stress and investigated the interactions between biotic and abiotic stresses through physiological analysis and quantitative fluorescence analysis of related genes under the corresponding stress conditions. This research is expected to lay a foundation for an indepth understanding of the molecular mechanisms underlying environmental responses in rice.

\section{ACKNOWLEDGMENTS}

Research supported by the Special Scientific Research Funds for Commonweal Section (Agriculture) (\#201203029, \#201003016), the China Rice Industry System Project (\#20112015) Special Funding for Basic Scientific Research and Operating Expense for Central Scientific Research Institutions of Commonwealth Section (\#2012RG004-2).

\section{Supplementary material}

\section{REFERENCES}

Abbruscato P, Nepusz T, Mizzi L, Del Corvo M, et al. (2012). OsWRKY22, a monocot WRKY gene, plays a role in the resistance response to blast. Mol. Plant Pathol. 13: 828-841.

Atkinson NJ and Urwin PE (2012). The interaction of plant biotic and abiotic stresses: from genes to the field. J. Exp. Bot. 63: 3523-3543.

Bagnaresi P, Biselli C, Orru L, Urso S, et al. (2012). Comparative transcriptome profiling of the early response to Magnaporthe oryzae in durable resistant vs. susceptible rice (Oryza sativa L.) genotypes. PLoS One 7: e51609.

Baisak R, Rana D, Acharya PBB and Kar M (1994). Alterations in the activities of active oxygen scavenging enzymes of wheat leaves subjected to water stress. Plant Cell Physiol. 35: 489-495.

De Datta SK (1981). Principles and Practices of Rice Production. International Rice Research Institute, Los Baños.

Dean RA, Talbot NJ, Ebbole DJ, Farman ML, et al. (2005). The genome sequence of the rice blast fungus Magnaporthe grisea. Nature 434: 980-986.

Derksen H, Rampitsch C and Daayf F (2013). Signaling cross-talk in plant disease resistance. Plant Sci. 207: 79-87.

Du B, Zhang W, Liu B, Hu J, et al. (2009). Identification and characterization of Bph14, a gene conferring resistance to brown planthopper in rice. Proc. Natl. Acad. Sci. U. S. A. 106: 22163-22168. 
Fujita M, Fujita Y, Noutoshi Y, Takahashi F, et al. (2006). Crosstalk between abiotic and biotic stress responses: a current view from the points of convergence in the stress signaling networks. Curr. Opin. Plant Biol. 9: 436-442.

Fujita Y, Fujita M, Yamaguchi-Shinozaki K and Shinozaki K (2009). Transcription Factors Involved in the Crosstalk Between Abiotic and Biotic Stress-Signaling Networks. In: Signal Crosstalk in Plant Stress Responses (Yoshioka K and Shinozaki K, eds.). Wiley-Blackwell, Oxford, 43-58.

Golldack D, Luking I and Yang O (2011). Plant tolerance to drought and salinity: stress regulating transcription factors and their functional significance in the cellular transcriptional network. Plant Cell Rep. 30: 1383-1391.

Gupta PC and O’Toole J (1986). Upland Rice: A Global Perspective. International Rice Research Institute, Los Baños.

Hadiarto T and Tran LS (2011). Progress studies of drought-responsive genes in rice. Plant Cell Rep. 30: 297-310.

$\mathrm{Hu}$ H, You J, Fang Y, Zhu X, et al. (2008). Characterization of transcription factor gene SNAC2 conferring cold and salt tolerance in rice. Plant Mol. Biol. 67: 169-181.

Jiang Y, Cai Z, Xie W, Long T, et al. (2012). Rice functional genomics research: progress and implications for crop genetic improvement. Biotechnol. Adv. 30: 1059-1070.

Khush GS (2001). Challenges for meeting the global food and nutrient needs in the new millennium. Proc. Nutr. Soc. 60: $15-26$.

Li C (2008). Automating dChip: toward reproducible sharing of microarray data analysis. BMC Bioinformatics 9: 231.

Li ZK and Xu JL (2007). Breeding for Drought and Salt Tolerant Rice (Oryza sativa L.): Progress and Perspectives. In: Advances in Molecular Breeding Toward Drought and Salt Tolerant Crops (Jenks MA, Hasegawa PM and Jain SM, eds.). Springer, Dordrecht, 531-564.

Liu W, Liu J, Ning Y, Ding B, et al. (2013). Recent progress in understanding PAMP- and effector-triggered immunity against the rice blast fungus Magnaporthe oryzae. Mol. Plant 6: 605-620.

Livak KJ and Schmittgen TD (2001). Analysis of relative gene expression data using real-time quantitative PCR and the 2(-Delta Delta C(T)) method. Methods 25: 402-408.

Luo LJ (2010). Breeding for water-saving and drought-resistance rice (WDR) in China. J. Exp. Bot. 61: 3509-3517.

Nguyen HT, Babu RC and Blum A (1997). Breeding for drought resistance in rice: physiology and molecular genetics considerations. Crop Sci. 37: 1426-1434.

Peng X, Hu Y, Tang X, Zhou P, et al. (2012). Constitutive expression of rice WRKY30 gene increases the endogenous jasmonic acid accumulation, PR gene expression and resistance to fungal pathogens in rice. Planta 236: 1485-1498.

Rabello AR, Guimaraes CM, Rangel PH, da Silva FR, et al. (2008). Identification of drought-responsive genes in roots of upland rice (Oryza sativa L.). BMC Genomics 9: 485.

Ren ZH, Gao JP, Li LG, Cai XL, et al. (2005). A rice quantitative trait locus for salt tolerance encodes a sodium transporter. Nat. Genet. 37: 1141-1146.

Ribot C, Hirsch J, Balzergue S, Tharreau D, et al. (2008). Susceptibility of rice to the blast fungus, Magnaporthe grisea. J. Plant Physiol. 165: 114-124.

Sharma R, Mohan Singh RK, Malik G, Deveshwar P, et al. (2009). Rice cytosine DNA methyltransferases - gene expression profiling during reproductive development and abiotic stress. FEBS J. 276: 6301-6311.

Shen X, Liu H, Yuan B, Li X, et al. (2011). OsEDR1 negatively regulates rice bacterial resistance via activation of ethylene biosynthesis. Plant Cell Environ. 34: 179-191.

Song WY, Wang GL, Chen LL, Kim HS, et al. (1995). A receptor kinase-like protein encoded by the rice disease resistance gene, Xa21. Science 270: 1804-1806.

Tirado MC, Clarke R, Jaykus LA, McQuatters-Gollop A, et al. (2010). Climate change and food safety: a review. Food Res. Int. 43: 1745-1765.

Ueno D, Yamaji N, Kono I, Huang CF, et al. (2010). Gene limiting cadmium accumulation in rice. Proc. Natl. Acad. Sci. U. S. A. 107: 16500-16505.

Valent B, Farrall L and Chumley FG (1991). Magnaporthe grisea genes for pathogenicity and virulence identified through a series of backcrosses. Genetics 127: 87-101.

Wang D, Pan Y, Zhao X, Zhu L, et al. (2011). Genome-wide temporal-spatial gene expression profiling of drought responsiveness in rice. BMC Genomics 12: 149.

Wei T, Ou B, Li J, Zhao Y, et al. (2013). Transcriptional profiling of rice early response to Magnaporthe oryzae identified OSWRKYs as important regulators in rice blast resistance. PLoS One 8: e59720.

Wilson RA and Talbot NJ (2009). Under pressure: investigating the biology of plant infection by Magnaporthe oryzae. Nat. Rev. Microbiol. 7: 185-195.

Xue D, Chen M and Zhang G (2009). Mapping of QTLs associated with cadmium tolerance and accumulation during seedling stage in rice (Oryza sativa L.). Euphytica 165: 587-596.

Yang DL, Yang Y and He Z (2013). Roles of plant hormones and their interplay in rice immunity. Mol. Plant 6: 675-685. Yang L, Su N, Wu M, Wang C, et al. (2011). Proteomics to identify pathogenesis-related proteins in rice roots under water 
deficit. Biologia 66: 477-483.

Yang S, Vanderbeld B, Wan J and Huang Y (2010). Narrowing down the targets: towards successful genetic engineering of drought-tolerant crops. Mol. Plant 3: 469-490.

Yang Z, Wu Y, Li Y, Ling HQ, et al. (2009). OsMT1a, a type 1 metallothionein, plays the pivotal role in zinc homeostasis and drought tolerance in rice. Plant Mol. Biol. 70: 219-229. 